\title{
Estado del arte de la plicatura de rectos
}

\author{
State-of-art of rectus shealth plication
}

\author{
Esteban Elena Scarafoni' ${ }^{1}$ Armando L. Pomerane², Carlos E. Sereday ${ }^{3}$
}

\section{RESUMEN}

Introducción. La abdominoplastia es un procedimiento que se utiliza para definir el contorno corporal actuando sobre todas las capas de la pared mediante la resección del exceso de piel y grasa subcutánea y la reparación del sistema músculo-aponeurótico. Uno de los componentes a tratar en toda abdominoplastia es la diastasis abdominal, que se logra a través de la plicatura de rectos. Muchas controversias han surgido acerca de la efectividad y duración de la corrección, del mejor tipo de sutura y las consecuencias sobre la presión intraabdominal y el embarazo.

Materiales y metodos. Se llevó a cabo una revisión sistemática de la literatura utilizando las bases de datos MEDLINE, LILACS, MEDES y SciELO. Tanto artículos de habla inglesa como hispana fueron tenidos en cuenta. Las palabras clave utilizadas para la búsqueda inicial fueron rectus plication, rectus shealth plication, diastasis, abdominal closure, plicatura abdominal.

Resultados. Luego de la búsqueda, 58 artículos fueron seleccionados y revisados. En base a la información obtenida, se dividió la información y se desarrollaron de manera objetiva en puntos separados.

Conclusiones. A pesar de que la bibliografía muestra conclusiones disímiles y variables, la plicatura es un procedimiento seguro y potencialmente beneficioso para la salud tanto en dolores lumbares como incontinencia urinaria, y puede usarse en aquellos casos intratables. Tanto suturas de reabsorción lenta como el PDS y las suturas no absorbibles pueden ser utilizadas para el cierre de la diastasis abdominal con excelentes resultados a largo plazo y sin riesgo de recurrencia.

Palabras claves: diastasis, abdominoplastia, plicatura abdominal.

\begin{abstract}
Introduction. Abdominoplasty is a body-contouring procedure for functional and aesthetic improvement that addresses deformity from excess skin and fat and musculofascial laxity. Diastasis repair by plication of the rectus sheath is an integral part of most abdominoplasty procedures. There is no consensus regarding what suture material is best and contradictory information about long term durability, changes in the intraabdominal pressure and pregnancy is seen in the literature.

Material and methods. A systematic review of current available literature was performed using the MEDLINE, LILACS, MEDES and SciELO databases. Key words used for initial data bank searches included "rectus plication", "rectus shealth plication" "diastasis" "abdominal closure", "plicatura abdominal". Furthermore, bibliographies and individual plastic surgery journals were searched for additional reference information.

Results. Fifty-eight articles met the criteria and were analyzed critically for inclusion. From this information, a narrative synthesis of data was undertaken.

Conclusions. Although current literature shows different conclusions, rectus shelth plication can be consider a safe and pottencially benefitial procedure with significant improvements in posture and quality of life. Absorbable and non absorbable sutures seems to be reliable sutures for the correction of rectus diastasis.
\end{abstract}

Key words: diastasis, rectus shealth plication, abdominoplasty.

REVISTA ARGENTINA DE CIRUGÍA PLÁSTICA 2020;26(1):23-30. HTTPS://DOI.ORG/10.32825/RACP/202001/0023-0030

\section{INTRODUCCIÓN}

La abdominoplastia es un procedimiento que se utiliza para definir el contorno corporal actuando sobre todas las capas de la pared mediante la resección del exceso de piel y grasa subcutánea y la reparación del sistema músculo-aponeurótico ${ }^{1}$. Según las últimas estadísticas de la ASPS, la abdominoplastia se encuentra entre las cinco cirugías mas realizadas en el año 2017 con un aumento del $107 \%$ desde el $2000^{2}$.

Uno de los componentes a tratar en toda abdominoplastia es la diastasis abdominal ${ }^{3}$. Esto se logra a través de la plicatura de rectos, que busca re-colocar los músculos en la línea media restaurando así la silueta.

Desde 1967, cuando Pitanguy ${ }^{4}$ describió la plicatura

1. Residente de Cirugía Plástica

2. Subjefe del Unidad del Servicio de Cirugía Plástica

3. Jefe de Unidad del Servicio de Cirugía Plástica

Hospital de Quemados del Gobierno de la Ciudad de Buenos Aires

$\triangle$ Correspondencia: Esteban Elena Scarafoni. Entre Ríos 663, B1636GAA Olivos, Provincia de Buenos Aires, Rep. Argentina. Tel...+54 9011155 9072890. estebanelenascarafoni@gmail.com

Los autores no declaran conflictos de intereses

Recibido: 22/04/2019 / Aceptado: 03/09/2019 vertical de la linea media con suturas no absorbibles, diferentes técnicas fueron utilizadas para la reparación de la diastasis ${ }^{5-8}$.

Muchas controversias han surgido acerca de la efectividad y duración de la corrección, el mejor tipo de sutura y las consecuencias sobre la presión intraabdominal y el embarazo.

El objetivo de este trabajo es realizar una revisión bibliográfica acerca de la plicatura vertical de los rectos reforzando los conceptos mas importantes.

\section{MATERIALES Y MÉTODOS}

Se llevó a cabo una revisión sistemática de la literatura utilizando las bases de datos MEDLINE, LILACS, MEDES y SciELO. Tanto artículos de habla inglesa como hispana fueron tenidos en cuenta. Las palabras clave utilizadas para la búsqueda inicial fueron rectus plication, rectus shealth plication, diastasis, abdominal closure, plicatura abdominal.

Los títulos y resúmenes fueron leídos, evaluados y seleccionados según la información contenida y los duplicados fueron descartados. Se excluyeron todos aquellos artículos de plicatura en pacientes posbariátricos. Una vez seleccionados los resúmenes, se buscaron los textos completos y se evaluaron las referencias de cada 


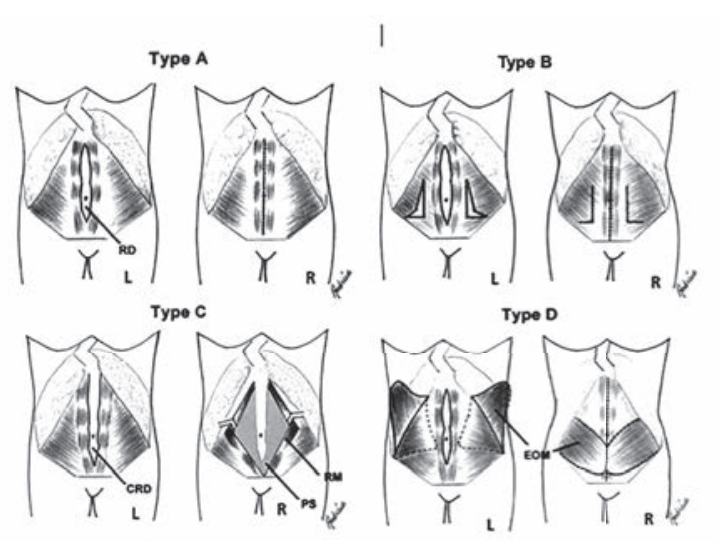

Figura 1. Se observan los diferentes tipos de deformidad de la pared mioaponeurótica y su tratamiento de acuerdo a la clasificación de Nahas. RD: Rectus plication, CRD: Congenital rectus diastasis, PS: Posterior shealth, EOM: external oblique muscle. Tomado de: Nahas, F. X. An aesthetic classification of the abdomen based on the myoaponeurotic layer. Plast Reconstr Surg 2001;108:1787.

artículo para obtener un número mayor. Por último, se buscaron artículos adicionales en las principales revistas de Cirugía Plástica.

\section{RESULTADOS}

Luego de la búsqueda, 58 artículos fueron seleccionados y revisados. En base a la información obtenida, se dividió la misma en 6 apartados y se desarrolló de manera objetiva.

\section{DISCUSIÓN}

\section{ANATOMÍA}

La plicatura de rectos es un paso casi mandatorio durante la abdominoplastia. Sin embargo, poco hay escrito acerca de la distancia normal entre los rectos y la anatomía de la línea alba.

En los tratados de Anatomía normal', se da el nombre de línea blanca o alba a una lámina fibrosa que ocupa en la línea media el espacio comprendido entre los músculos rectos y que se forma por el entrecruzamiento de las diferentes hojas aponeuróticas de los tendones de inserción de los músculos oblicuo mayor, menor y transverso. Según Testut ${ }^{10}$, la línea alba mide sucesivamente de anchura 5-6 mm en su parte superior 10-12 $\mathrm{mm}$ a nivel medio, $20-22 \mathrm{~mm}$ a nivel umbilical y $2-3$ $\mathrm{mm}$ por debajo del ombligo.

Rath $^{11}$, en 1996, publicó un estudio anatomo-radiológico de la pared abdominal para determinar las medidas estándares. Para este fin, realizó una medición de la línea alba en 40 cadáveres ( 20 hombre y 20 mujeres), con una media de edad de 83 años (entre 62 y 99) en tres niveles: a nivel umbilical, en un punto medio entre el ombligo y el apéndice xifoides y a una medida equidistante entre el ombligo y el pubis. El ancho medio de la línea alba fue de $1,72 \mathrm{~cm}$ por encima $(0,5-3,5), 2,25$

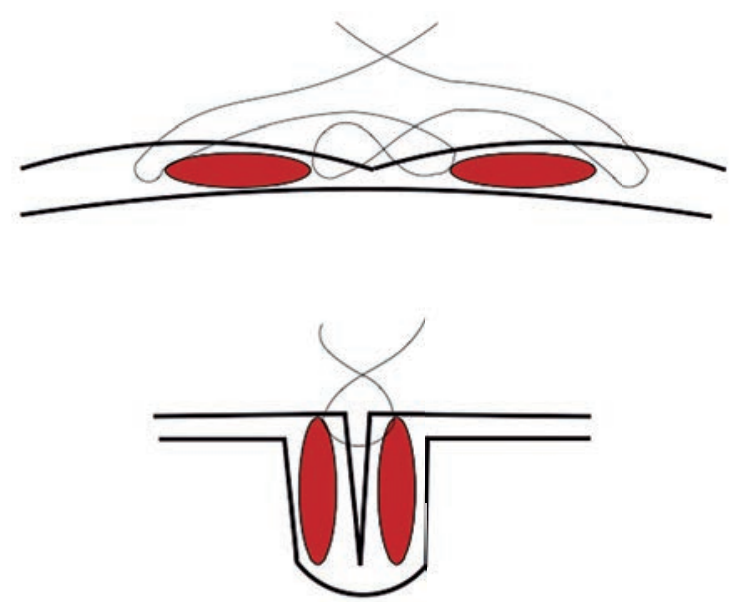

Figura 2. Representación del WARP (wide abdominal rectus plicaction). Se observan dos cortes axiales que ilustran la sutura desde los bordes externos de los músculos rectos para girar los mismos 90 grados

$\mathrm{cm}$ a nivel del ombligo $(0,5-5)$ y de $0,66 \mathrm{~cm}$ por debajo del mismo $(0,1-2)$. No hubo diferencia significativa entre edad y sexo.

A su vez, realizó un estudio in vivo en 40 pacientes (16 mujeres y 24 hombres con una edad media de 51,5 años) a quienes se les realizó una tomografía axial computarizada, mostrando valores superiores a los cadavéricos y con una diferencia entre edades y sexos. Con respecto al sexo, el estudio mostró un diferencia de $4 \mathrm{~mm}$ mayor en hombre a nivel supraumbilical. A su vez, mostró que el ancho de la línea alba se incrementaba significativamente con la edad excepto a nivel umbilical y estableció como punto de corte la edad de 45 años. Así, definió como valores normales a nivel supra umbilical: 5-6 mm antes de los 45 años y 12-14 mm después de los 45; a nivel umbilical: $19-23 \mathrm{~mm}$ y a nivel infraumbilical: 5-6 mm antes de los 45 años y 9-11 mm a partir de esta edad.

A pesar de ser el único estudio con disecciones cadavéricas, la población estudiada fue heterogénea con una gran variabilidad de edades entre sexos y entre la población viva y la cadavérica. A su vez, no incluyó el IMC de los pacientes ni el número de embarazos previos en las mujeres.

Beer en $2009^{12}$ evaluó la distancia interrectal normal en 150 pacientes nulíparas entre 20 y 45 años con un IMC menor a $30 \mathrm{~kg} / \mathrm{m}^{2}$ con ultrasonido en tres puntos de referencia: en el origen del apéndice xifoide, 3 $\mathrm{cm}$ por encima y $2 \mathrm{~cm}$ por debajo del ombligo. Observó que la línea alba era desigual en ancho entre los puntos, siendo mayor la distancia $3 \mathrm{~cm}$ por encima del ombligo, seguido por debajo del mismo y por último a nivel del apendice xifoides. Concluyó que la distancia entre los rectos puede considerarse normal hasta $15 \mathrm{~mm}$, $22 \mathrm{~mm}$ y $16 \mathrm{~mm}$ a nivel del xifoides, supra- e infraumbilical, respectivamente. Además, no encontró diferencias significativas entre las diferentes edades. 
Brauman ${ }^{13}$ evaluó clínicamente 92 pacientes que luego se sometieron a una abdominoplastia en donde realizó mediciones intraoperatorias de la diastasis. Observó que la diastasis no está relacionada con la protrusión abdominal sino que esta se da por un estiramiento y laxitud de toda la pared anterolateral. Por lo tanto, el tamaño de la diastasis no es un indicador confiable de la protrusión abdominal y la presencia de laxitud abdominal y protrusión no es sinónimo de diastasis. Los abdómenes planos pueden tener diastasis muy marcadas y viceversa. A su vez, el sitio de mayor protrusión abdominal no se correlaciona siempre con el sitio de mayor diastasis.

En cuanto a la forma más confiable para medir la diastasis abdominal, Mendes ${ }^{14}$, en 2007, realizó un estudio para evaluar la efectividad de la ecografía como método diagnóstico. Para eso, 20 mujeres (19 de las cuales tuvieron cesárea) fueron evaluadas preoperatoriamente con ultrasonido, y luego la distancia de la distasis fue comparada con medidas obtenidas en el intraoperatorio por dos evaluadores independientes con un compás. Las medidas fueron realizadas a 3, 6, 9 y $12 \mathrm{~cm}$ del borde superior del ombligo, a 2 y $4 \mathrm{~cm}$ por debajo del mismo y a nivel umbilical. Concluyeron que no había cambios estadísticos significativos entre las medidas tomadas con ecografía y las intraoperatorias por encima y a nivel umbilical, pero sí a nivel infraumbilical, debido a la fibrosis producida por la cesárea.

Nahas ${ }^{15}$ obtuvo la misma conclusión pero evaluando la diastasis con tomografía y comparándola con las medidas intraoperatorias. Sin embargo, la mayor diferencia entre ambas medidas fue de $0,3 \mathrm{~cm}$, lo cual, desde el punto de vista clínico y quirurgico, no es relevante a pesar de ser estadísticamente significativo.

\section{CLASIFICACIÓN}

Varias clasificaciones fueron descriptas en abdominoplastia basadas en diferentes parámetros como laxitud cutánea, miofascial y cantidad de tejido graso ${ }^{16,17}$.

Sin embargo, la clasificación de Nahas de $2001^{18}$ apunta específicamente a la laxitud del complejo mioaponeurótico estableciendo diferentes grados de deformidad con su tratamiento consecuente. Él clasificó las deformaciones en 4 tipos en base a 88 pacientes (Figura 1).

Tipo A. Aquellas pacientes que presentan diastasis abdominal secundaria al embarazo con una cintura bien definida cuyo tratamiento es una plicatura vertical de la aponeurosis. Esta es la forma de presentación mas común.

Tipo B. Son aquellas pacientes que presentan diastasis secundaria al embarazo y además tienen laxitud en el área infraumbilical y lateral, lo cual se evidencia en el intraoperatorio por la presencia continua de laxitud luego de realizar la plicatura vertical. En esta caso, se debería adicionar una plicatura de oblicuo externo en "L".

Tipo C. Son aquellas pacientes que presentan inserción lateral congénita de los músculos rectos. En este caso, la simple plicatura vertical de los mismos genera- ra una recidiva, con lo cual deberían avanzarse los músculos despegándolos de la hoja posterior de la vaina.

Tipo D. Son aquellas pacientes que presentan una diastasis de recto y una cintura mal definida. En este caso, además de la plicatura vertical, es necesario un avance del oblicuo mayor ${ }^{19}$. Son las menos frecuentes.

\section{BENEFICIOS EN LA SALUD}

Fue Toranto ${ }^{20,21}$ el primero en utilizar la abdominoplastia como tratamiento para los dolores lumbares crónicos intratables. La modificación que le permitió esto fue la plicatura amplia entre los bordes externos de los rectos generando una rotación de los mismos 90 grados y un tensado superior de la pared abdominal al que el llamó WARP (wide abdominal rectus plication) (Figura 2). Demostró en 24 de 25 pacientes una mejoría evidenciada por una disminución inmediata y sostenida del dolor lumbar y un aumento de los espacios intervertebrales y los ángulos lumbosacros en la resonancia magnética y las radiografías de abdomen, respectivamente. Esto se explica por el modelo propuesto por Gratovetsky ${ }^{22}$ y otros autores ${ }^{23}$ que establece que la contracción de los músculos laterales del abdomen (principalmente oblicuo interno y transverso) tensan la fascia toracolumbar a la que se encuentran unidos. Esta actúa como un ligamento pasivo para la columna disminuyendo la acción de los músculos lumbares y así la presión sobre los discos intervertebrales. A su vez, cambia el centro de gravedad del individuo hacia adelante mejorando la lordosis y los ángulos lumbosacros. La complicación de este procedimiento es el aumento excesivo de la presión intraabdominal. Ello puede generar alteraciones respiratorias graves como ocurrió en 3 pacientes que incluyeron, uno con edema pulmonar agudo y otro con insuficiencia ventilatoria aguda que requirió respirador por varios días.

Oneal ${ }^{24}$, en 2011, presentó también una mejoría inmediata y duradera en 8 pacientes tratados con la técnica WARP. A diferencia de Toranto, en 5 pacientes se realizó una plicatura de los bordes laterales del recto con suturas irreabsorbibles, en 1 se realizó la plicatura a nivel del tercio lateral del músculo recto y en los restantes dos a nivel del punto medio del músculo, sin mostrar diferencias en la mejoría. La presión fue monitoreada a través de un catéter femoral y de una sonda vesical evitando un aumento por encima de 8 y $15 \mathrm{~cm}$ de agua, respectivamente. A pesar de que ninguno superó los valores preestablecidos, uno de los pacientes intercurrió con un íleo y otro con dificultad respiratoria, los cuales se autolimitaron en el transcurso del primer dia. A pesar de que a mayor plicatura mayor tensado de la pared, y por ende de la fascia toracolumbar, la plicatura de los bordes internos de los rectos también generaría una mejora en el dolor lumbar como lo demuestra Temel ${ }^{25}$, en un estudio prospectivo de 40 pacientes sometidas a abdominoplastia con plicatura de rectos convencional, donde observó una disminución del do- 
lor lumbar evaluada con cuestionarios validados y una mejoría de la lordosis lumbar y de los ángulos lumbosacros evidenciado con radiografías mediante la técnica de Cobb. Ninguno de las pacientes sufrió complicaciones por aumento de presión abdominal a los 6 meses posoperatorio.

La abdominoplastia no solo mejora el dolor lumbar sino también la incontinencia urinaria. Los primeros artículos al respecto publicados fueron reportes de un caso en mujeres de mediana edad que presentaron disminución subjetiva de los síntomas ${ }^{26-28}$. En estos artículos, la hipótesis en la mejora estaba relacionada con el aumento de la presión intraabdominal generada por la plicatura de rectos, que mejoraría la presión de vaciado vesical.

Solanki ${ }^{29}$, que también pensaba en el mismo mecanismo, realizó un estudio retrospectivo en 100 pacientes operadas de abdominoplastia de las cuales 46 respondieron a un cuestionario creado por el autor; 27 (59\%) refirieron incontinencia urinaria preoperatoria y, de estas, 7 (26\%) mostraron una mejora luego de 6 meses de cirugía. También evidenció que el parto vaginal era un factor predictivo para la incontinencia urinaria preoperatoria.

Carruthers ${ }^{30}$ hizo lo propio y realizó un estudio retrospectivo en 100 pacientes que fueron sometidos a abdominoplastia de los cuales 50 reportaron incontinencia urinaria preoperatoria. Un 60\% (30 pacientes) mostró una mejora de los síntomas a largo plazo y, de ese número, un $53 \%$ mostró un alivio definitivo de la incontinencia. Carruthers explica que un factor por el que mejoraría la incontinencia seria el tensado de la fascia de Scarpa, la cual es continuación de la fascia de Colles a nivel perineal y esto generaría un alargamiento y reposición de la uretra. Ambos trabajos, si bien demostraron una mejora de la incontinencia, fueron trabajos retrospectivos que utilizaron cuestionarios no validados y con un número bajo de pacientes.

En un estudio prospectivo y multicéntrico realizado en 2018 en Australia, liderado por Taylor ${ }^{31}$, se evidenció una mejoría estadísticamente significativa tanto de la incontinencia urinaria como el dolor de espalda crónico en 214 pacientes. En cuanto a los datos demográficos, tenían una edad media de 42,1 años, con un índice de masa corporal (IMC) de $26,3 \mathrm{~kg} / \mathrm{m}^{2}$ y una media de 2,5 hijos. Para la evaluación de la incontinencia urinaria y del dolor de espalda se utilizaron dos cuestionarios internacionales validados que se compararon en el pre- y posoperatorio a las 6 semanas y a los 6 meses. Cinco tipos diferentes de abdominoplastia fueron utilizados sin mostrar diferencia significativa entre la cirugía empleada y el grado de beneficio a los 6 meses. En cuanto a la incidencia de dolor de espalda crónico, se evidenció que solo un 8,8\% (19 pacientes) no presentaban dicho síntoma y que los factores predictivos significativos eran un IMC mayor a $25 \mathrm{~kg} / \mathrm{m}^{2}$ y la presencia de hernias umbilicales.
En cuanto a la incontinencia urinaria, 27,5\% (59 pacientes) no la presentaban preoperatoriamente y de las que sí, los factores predictivos fueron la edad mayor a 40 y los partos vaginales.

A pesar de no poseer grupo control y de no emplear la misma técnica entre los cirujanos, el hecho de ser un estudio prospectivo con una gran muestra lo vuelve un estudio confiable.

\section{TIPO DE SUTURA}

Ha habido un largo debate entre el uso de suturas absorbibles $v$ s. no absorbibles para la corrección de la diastasis. Las primeras tienen la ventaja de no generar induraciones palpables a través de la piel en pacientes delgadas y un menor riesgo de granulomas, extrusion e infección. Sin embargo, la corrección a largo plazo con dicha sutura es cuestionable debido a las presiones a las que está sometida la línea alba, como las contracciones musculares, la retracción cicatrizal, la elasticidad de los tejidos y los aumentos de la presión intraabdominal causada por los propios órganos, las actividades físicas o condiciones como la obesidad.

El primero en realizar un estudio comparativo entre suturas para la corrección de la distasis abdominal fue Birdsell ${ }^{32}$, en 1981, quien comparó en 30 pacientes el grado de corrección a los 6 meses con el uso de ácido poliglicólico $2.0 \mathrm{vs}$. nylon 2.0. La edad de la muestra era entre 27 y 42 años y el ancho de la diastasis era entre 4 y $6 \mathrm{~cm}$. Luego de realizar una abdominoplastia convencional, utilizó un doble plano de sutura con puntos separados primero y luego una sutura continua. Para valorar el grado de corrección, colocó clips metálicos a cada lado de la plicatura a 1 y 3 $\mathrm{cm}$ por debajo y por encima del ombligo y comparó la separación de los mismos en radiografías abdominales seriadas tomadas de pie a las 48 horas y a los 6 meses posoperatorios. A su vez, en dos pacientes les realizó radiografías al año. Demostró que no hubo diferencias en la separación de los clips usando una sutura absorbible frente a una irreabsorbible. A pesar de ser un estudio comparativo, tiene la desventaja de contar con una muestra pequeña, con seguimiento a corto plazo y con un método operador dependiente como es la radiografía.

En 2001, Nahas ${ }^{15}$ realizó un estudio comparando la corrección de la diastasis utilizando Nylon 2,0 vs. polidioxanona 0 en 20 pacientes divididos en 2 grupos de 10 cada uno. La edad media era de 32,5 y 38,5 años en cada grupo, respectivamente. Se realizó una plicatura de rectos en dos planos: un primer plano con puntos separados cada $0,4 \mathrm{~cm}$ y otro más superficial con sutura continua. Se compararon las correcciones mediante cortes tomográficos realizados preoperatoriamente, a las 3 semanas y a los 6 meses posoperatorios en dos puntos: $3 \mathrm{~cm}$ por encima del ombligo y $2 \mathrm{~cm}$ por debajo del mismo. La corrección de la diastasis se mantuvo a los 6 meses en ambos grupos sin diferencias estadís- 
ticas significativas. A pesar de que la muestra es baja y el seguimiento corto, el método utilizado es más fidedigno.

En 2001, Van Uchelen ${ }^{33}$ realizó un estudio retrospectivo en 40 pacientes en los que se reparó la diastasis con el uso de suturas absorbibles (Vycril 0, 2.0 o Prolene). La edad media de los pacientes era de 46 años con un tiempo posoperatorio promedio de 64 meses. Se observó en 16 pacientes (40\%) una recurrencia de la diastasis. A pesar del tiempo posoperatorio más alejado y del mayor número de pacientes en la muestra, este estudio presenta varios aspectos criticables. En primer lugar, el estudio es retrospectivo y no hay imagen preoperatoria o posoperatoria inmediata que permita comparar si la recurrencia fue producto de una reparación inadecuada o del uso de la sutura. Además, el tipo de sutura utilizado no está especificado en cada caso nombrando varios tipos incluyendo Vycril 0, Vycril 2, 3 o 4.0, Prolene y Mersilene como posibles responsables. En segundo lugar, los procedimientos quirúrgicos no fueron realizados por el mismo cirujano. Incluso, aclara, en varias ocaciones fue un residente el que realizó la cirugía. En tercer lugar, 6 de las 16 pacientes que tuvieron recurrencia de la diastasis tuvieron un embarazo en el posopertorio. Por último, habla de una sutura paramediana y no de aproximación de los bordes mediales de los rectos, lo cual hace suponer el exceso de tensión puesto en las suturas.

En 2005, Nahas ${ }^{34}$ realizó otro estudio prospectivo para evaluar la eficacia a largo plazo de la corrección de la diastasis utilizando Nylon 2.0. En este estudio, la muestra fue de 12 pacientes de entre 18 y 52 años sometidas a abdominoplastia, en las que se utilizó para la sutura de los rectos Nylon 2.0 en dos planos. Al igual que en su estudio anterior, utilizó la tomografía como método de comparación a las 3 semanas, 6 meses y luego en un tiempo posoperatorio a largo plazo de 81,2 meses en promedio y hasta 7 años. En ninguno de los casos hubo recurrencia de la diastasis.

Elkhatib $^{35}$, en 2011, realizó un estudio prospectivo valorando la recurrencia a largo plazo de las suturas no absorbibles utilizadas. En este caso, utilizó la RMN como método de control por ser operador independiente y no generar radiación. El número de la muestra era de 20 pacientes con una edad promedio de 33,6 años (entre 27 y 54), a quienes se les realizó una lipoabdominoplastia con una plicatura en un plano continuo utilizando Nylon 0. Se compararon los resultados de la resonancia magnética del preoperatorio y del posoperatorio entre 6 y 25 meses sin evidenciar recurrencia. En otro estudio realizado por Tadiparthi en $2011^{36}$, no se evidencia recurrencias en la distasis abdominal en estudios ecográficos realizados a los 3,6 y 12 meses posoperatorios en 28 pacientes. La edad promedio de los mismos era de 36 años y se realizó plicatura con una sutura continua de Nylon 0. Lo particular de este estudio es que, a pesar de que la muestra no era alta, in- cluyó tanto pacientes multíparas y nulíparas y pacientes con factores de riesgo pulmonares como pacientes asmáticas y con EPOC, sin mostrar una recurrencia. Rosen $^{37}$, en 2011, publicó un artículo retrospectivo de 34 pacientes operadas de abdominoplastia a quienes se les realizó una plicatura en dos planos con polidioxanona 0 en 18 pacientes y sutura barbada de PDO en las restantes. La edad media de la muestra era de 43,6 años (entre 33 y 67). Se realizó un control clínico a los 3 meses, 6 meses y al año de la cirugía por dos examinadores, sin evidencia de recurrencia. A pesar de ser uno de los únicos artículos que utiliza sutura barbada, este estudio tiene la desventaja de ser retrospectivo y de no tener un método de control imagenológico comparable. En otro estudio de Nahas ${ }^{38}$, en 2011, utilizando sutura en dos planos con polidioxanona 0 en 12 pacientes sometidas a abdominoplastia con edad media de 38,6 años, no se evidencia recurrencia en tomografías realizadas a las 3 semanas y a los 40 meses.

Mestak39, en 2012, realizó un estudio de casos y controles para valorar la eficacia en el uso de suturas reabsorbibles a largo plazo. El grupo de casos contenía 51 pacientes, con una mediana de edad de 41 años (entre 25 y 64) a quienes se les realizó una abdominoplastia con plicatura de rectos con una sutura continua de polidioxanona 0 y se valoró la corrección con ultrasonido en tres sitios: a nivel equidistante entre el xifoides y el ombligo, justo por encima del ombligo y a nivel equidistante entre el ombligo y el pubis entre los 12 y los 41 meses posoperatorios (media 20,8). Se comparó dicho resultado con un grupo control de 10 mujeres nulíparas con edad media de 26 años. Se demostró que no había diferencias estadísticas significativas entre los grupos y que ninguna paciente mostró recurrencia a largo plazo. A pesar de que las edades entre los dos grupos era heterogénea, el hecho de ser prospectivo, con un follow up de más de un año en promedio con 51 pacientes, y que utilizó la misma sutura por el mismo cirujano lo vuelve un estudio confiable. Además, a pesar de utilizar la ecografía como método de control, esta fue realizada por el mismo médico.

De Castro ${ }^{40}$, en 2013, realizó un estudio retrospectivo en 38 pacientes comparando ecografías pre- y posoperatorias de un grupo de pacientes operados al año y otro grupo a los cinco años de la cirugía. Todos fueron operados siguiendo la técnica de Pitanguy con sutura en X invertida con Nylon 2.0 para la plicatura de los rectos. Evidenció un 5,3\% de recurrencia (2 pacientes de 38). Ambos pacientes pertenecían al grupo de control posoperatorio corto, sin mostrar recurrencias en el largo plazo. Esto lo asoció a que los pacientes tenían en el preoperatorio una inserción lateral de los músculos rectos (Grupo Nahas C), lo cual, tal y como lo explica Nahas, hubiese requerido otro tratamiento.

En 2017, Gama ${ }^{41}$ realizó un estudio prospectivo comparando tres grupos: el primero, en donde se realizó una doble sutura con Nylon 2.0; el segundo, en don- 
de se realizó una sutura continua simple con Nylon 2.0, y un tercer grupo con la utilización de sutura de Nylon barbada. Se calculó el tiempo total de la cirugía y el tiempo de la corrección de diastasis y la valoración de la corrección a los 6 meses con ecografía comparada con la del preoperatorio, todas realizadas por el mismo individuo. La edad media era 36,8 años y el IMC medio era de 24,21, con un total de 30 pacientes. Se llegó a la conclusión de que tanto si realizaba uno o dos planos con Nylon, la recurrencia a los 6 meses era nula. Sin embargo, el tiempo intraoperatorio era menor en el primer caso con una diferencia significativa. A su vez, el grupo en donde se utilizó sutura barbada, presentó un $30 \%$ de recurrencia sin mostrar una disminución significativa en el tiempo comparada con el grupo 2 .

\section{CORRECCION DE DIASTASIS Y PRESIÓN INTRAABDOMINAL}

La tensión generada por la plicatura vertical aumenta la presión intraabdominal, lo cual puede predisponer a un síndrome de compresión con alteraciones urinarias, ventilatorias y un aumento del riesgo de trombosis venosa profunda y por ende de tromboembolismo pulmonar.

Hay artículos reportados en la literatura de síntomas de aumento de presión intraabdominal luego de realizar una dermolipectomía con plicatura de rec$\operatorname{tos}^{42-44}$, como alteraciones respiratorias y reflujo gastroesofágico que, en algunos casos, requirieron la liberación de las suturas. Sin embargo, cabe destacar que en estos artículos se realizaban plicaturas amplias de hasta $13 \mathrm{~cm}$.

Uno de los primeros en medir y comparar las presiones intraabdominales fue Losken ${ }^{45,46}$, quien realizó una comparación de las mismas entre pacientes sometidos a TRAM para reconstrucción mamaria. Realizó un estudio prospectivo en 44 pacientes que fueron agrupados según el tipo de cierre de la zona dadora: TRAM unilateral con cierre simple, TRAM bilateral con cierre simple y aquellos en los que se realizó un cierre con malla. Se concluyó que a mayor tensión de cierre, mayor presión intraabdominal y mayor riesgo de generar alteraciones respiratorias y urinarias y de complicaciones locales. Este aumento de la presión fue transitorio y disminuyó a partir del segundo día.

$\mathrm{Al} \mathrm{Basti}{ }^{47}$, en 2004, realizó un estudio prospectivo en 43 pacientes multíparas y obesas con un IMC medio de $36 \mathrm{~kg} / \mathrm{m}^{2}$ y una edad media de 38 años en donde se valoró la presión intraabdominal a través de una sonda vesical pre- y posplicatura. A su vez, realizó exámenes pulmonares preoperatorios y a los 2 meses posoperatorios para valorar la función respiratoria. Dieciocho de estas pacientes presentaban asma leve, sin utilización de corticoides. La corrección de la diastasis se realizó con Nylon 0 de forma continua tanto del recto como del oblicuo mayor. Demostró un aumento estadísticamente significativo de la presión intraab- dominal, pero sin repercusiones clínicas. Los valores pulmonares pre- $y$ posoperatorios no mostraron cambios significativos.

En 2006, Neto ${ }^{48}$ realizó un estudio prospectivo comparando los valores de presión intraabdominal previo a la cirugía, posterior a la misma y en el día 1 . Todas las pacientes tenían un IMC menor a 30 y una edad menor a 50 años. Se realizó la plicatura con Nylon 2.0 en forma continua y se valoró la presión a través de una sonda vesical. En todos los pacientes hubo un aumento de la presión intraabdominal luego de la plicatura, que disminuyó en las primeras 24 horas. La desventaja de este estudio es que no especifica en qué momento del posoperatorio se evalúa la presión ni si la paciente se encontraba con ropa compresiva o en posición semiflexionada, lo cual alteraría la medición y no se podría comparar con las del preoperatorio.

Huang ${ }^{49}$ comparó la presión intraabdominal en 12 pacientes sometidas a abdominoplastia con un grupo control de 10 pacientes sometidas a cirugía de reducción mamaria. Se realizó una abdominoplastia con plicatura vertical y oblicuas sin especificar el tipo de sutura y se tomaron las presiones intraabdominales por via vesical en el preoperatorio en posición horizontal y a 30 grados, posplicatura también en ambas posiciones, luego del cierre cutáneo y al siguiente día tanto a las pacientes sometidas a abdominoplastias que se encontraban con compresión y las de reducción que no. No había diferencias significativas en la edad, IMC y número de embarazos entre los grupos. Dos pacientes de los 12 eran fumadoras. Concluyó que la presión abdominal aumentaba con la semiflexión y con la plicatura de una forma estadísticamente significativa pero clínicamente neutra, que disminuyó en el primer día posoperatorio. A su vez, el uso de ropa compresiva en el posoperatorio aumentó la presión intraabdominal de manera significativa.

Vaca Escobar ${ }^{50}$, en 2007, evaluó en 18 pacientes las presiones abdominales pre- y posplicatura y realizó un estudio espirométrico preoperatorio a las 24 hs y a los 15 dias para valorar la función respiratoria. Estas pacientes tenían una edad promedio de 34,1 años, una paridad promedio de 2,4 e IMC promedio de 27.5. El promedio de plicatura de los rectos abdominales fue de $8,64 \mathrm{~cm}$. Evidenció un aumento en la presión abdominal luego de la plicatura de $4,46 \mathrm{~cm}^{2}$ promedio en el preoperatorio a $13,56 \mathrm{~cm}^{2}$ encontrándose diferencia significativa entre las dos, sin evidencias clínicas. A su vez, demostró que la capacidad vital forzada a las 24 hs fue significativamente menor que en el preoperatorio pero no a los 15 dias. Sin embargo, la capacidad vital disminuida por el proceso quirúrgico a los 15 días no se llegó a recuperar completamente hasta los valores iniciales, aunque sí se recuperó parcialmente (50\%). Mercedes $^{51}$, en 2009, en un estudio prospectivo de 100 pacientes sometidos a abdominoplastia, concluyó que se evidenció como una constante que la combina- 
ción de IMC elevado con una plicatura mayor de 10 $\mathrm{cm}$ provoca un aumento significativo de la presión intrabdominal, no resultando así cuando estas variables se presentaron por separado. A su vez, no hubo diferencias significativas entre realizar una plicatura vertical y una triple plicatura.

Un estudio prospectivo fue realizado en 2016 por Pereira et al..$^{52}$. En dicho estudio, 10 pacientes mayores de 18 años (media 43,5 ( con IMC menor a 35 (media 27,16) $\mathrm{y}$ sin antecedentes de tabaquismo o alteraciones pulmonares previas fueron incluidas en el estudio en donde se les realizaron las mediciones de la presión intraabdominal y la compliance pulmonar antes y después de la plicatura de rectos realizada con sutura continua de polipropileno 1. La presión intraabdominal aumentó de 6,6 $\mathrm{mmHg}$ a $9,3 \mathrm{mmHg}$ y la compliance pulmonar descendió de $38,97 \mathrm{ml} / \mathrm{cmH}_{2} \mathrm{O}$ a $36,5 \mathrm{ml} / \mathrm{cmH}_{2} \mathrm{O}$, ambos cambios estadísticamente significativos. Sin embargo, ninguno de los mismos logró cambios clínicos ni logró sobrepasar los valores patológicos.

En 2015, Rodriguez ${ }^{53}$ realizó un estudio prospectivo para valorar si el tamaño de la diastasis abdominal influía sobre la presión intraabdominal. Para ello, 17 pacientes con una media de edad de 31,9 y un IMC menor a 28 , sin antecedentes pulmonares patológicos, fueron sometidas a una abdominoplastia con plicatura abdominal en dos planos con Nylon 2.0. Se evaluó la presión intraabdominal a través de una sonda vesical previo y posterior a la plicatura, se midió el ancho de la distasis y se calculó según el volumen abdominal. Concluyó que a pesar de que la presión aumentó en todos los casos (sin sobrepasar los valores normales), no hubo relación con la magnitud de la distasis, sugiriendo como hipótesis que las personas con mayor diastasis tienen mayor laxitud musculocutánea con una mayor compliance abdominal.

\section{PLICATURA DE RECTOS Y EMBARAZO}

El embarazo es una de las principales causas de diastasis abdominal. Sin embargo, las consecuencias que tiene sobre una plicatura de rectos no está tan clara. Hay varios artículos que hablan acerca del embarazo sin riesgos posteriores a una abdominoplastia, incluso luego de dos meses 54-56. Sin embargo, hay pocos artícu- los en la literatura que muestran lo que ocurre con la plicatura luego del embarazo.

Los primeros estudios encontrados son acerca del embarazos posteriores a una reconstrucción mamaria con colgajo TRAM. Chen y Hartrampf ${ }^{57}$ publicaron sobre 600 casos, 6 pacientes que tuvieron embarazos sin consecuencias. El tiempo transcurrido entre el TRAM y el embarazo fue entre 12 y 46 meses y la forma de cierre fue en 5 de los casos en forma primaria y en un caso con malla. En ningún caso se observó diastasis ni hernias clínicamente evidenciables aunque ningún estudio imagenológico fue utilizado para la certificación. Llegaron a la conclusión de que el tiempo prudente para esperar al embarazo es de un año para dejar madurar la cicatriz.

Nahas ${ }^{58}$, en 2002, publicó el caso de una paciente de 25 años que se embarazó 2 años y medio luego de una abdominoplastia convencional con sutura de la diastasis con Nylon 4,0 en dos planos: uno con puntos separados enterrados con una distancia de $0,4 \mathrm{~cm}$ del otro y luego una sutura continua. Se realizó una TAC 15 meses luego del parto, la cual no mostró recurrencia de la diastasis evidenciada con una zona fibrótica entre ambos rectos. Nahas propone que esto se debe a que la línea alba no es extensible y que el aumento de la presión abdominal y la elasticidad se deben a la distensión de los músculos rectos que luego del embarazo, por acción contráctil de los mismos, vuelven a la normalidad sin alterar la línea. Él también recomienda al menos 1 año para que se genere una fibrosis que no genere recurrencia de la misma.

\section{CONCLUSIONES}

La plicatura de rectos es un paso esencial en toda abdominoplastia para restaurar el contorno corporal. A pesar de que la bibliografia muestra conclusiones disímiles y variables, la plicatura es un procedimiento seguro y potencialmente beneficioso para la salud tanto en dolores lumbares como incontinencia urinaria, y puede usarse como tratamiento en aquellos casos intratables. Tanto suturas de reabsorción lenta como el PDS y las suturas no absorbibles pueden ser utilizadas para el cierre de la diastasis abdominal sin riesgo de recurrencia a largo plazo.

\section{BIBLIOGRAFÍA}

1. Winocour, J., Gupta, V., Ramirez, R. Abdominoplasty: Risk Factors, Complication Rates, and Safety of Combined Procedures. Plast Reconst Surg. 2015. Volume 136, Number 5

2. https://www.plasticsurgery.org/documents/News/Statistics/2017/ plastic-surgery-statistics-full-report-2017.pdf

3. Netscher DT, Wigoda P, Spira M et al. Musculoaponeurotic plication in abdominoplasty: How durable are its effects? Aesthetic Plast Surg. 1995; 19:531-534.
4. Pitanguy I. Abdominal lipectomy: An approach to it through an analy sis of 300 consecutive cases. Plast Reconstr Surg. 1967;40:384-391

5. Yousif NJ, Lifchez SD, Nguyen HH. Transverse rectus sheath plication in abdominoplasty. Plast Reconstr Surg. 2004;114: 778-784.

6. Nahas FX, Ferreira LM, Mendes Jde A. An efficient way to correct recurrent rectus diastasis. Aesthetic Plast Surg. 2004; 28:189-196.

7. Ramirez OM. Abdominoplasty and abdominal wall rehabilitation: A comprehensive approach. Plast Reconstr Surg. 2000; 105:425-435. 
8. Abramo AC, Viola JC, Marques A. The H approach to abdominal muscle aponeurosis for the improvement of body contour. Plast Reconstr Surg. 1990;86:1008-1013

9. Latarjet M. y Ruíz Liard A. Anatomía Humana. Editorial Médica Panamericana. Barcelona (1993)

10. Testut L, Latarjet A.Tratado de anatomía humana. 9. ed. Barcelona, Salvat, 1960. V. 2

11. Rath AM, Attali P, Dumas JL, Goldlust D, Zhang J, Chevrel JP. The abdominal linea alba: an anatomo-radiologic and biomechanical study. Surg Radiol Anat 1996;18:281e8.

12. Beer GM, Schuster A, Seifert B, Manestar M, Mihic-Probst D, Weber SA. The normal width of the linea alba in nulliparous women. Clin Anat. Wiley Subscription Services, Inc., A Wiley Company; 2009 Sep;22(6):706-11.

13. Brauman, D. Diastasis Recti: Clinical Anatomy. Plast Reconst Surg. 2008. Volume 122, Number 5

14. Mendez, A, Nahas FX, Veiga DF, Mendes FV, Figueiras RG, Gomes HC, Ely PB, Novo NF, Ferreira LM. 2007. Ultrasonography for measuring rectus abdominis muscles diastasis. Acta Cir Bras 22:182-186.

15. Nahas FX, Augusto SM, Ghelfond C. Nylon versus polydioxanone in the correction of rectus diastasis. Plast Reconstr Surg. 2001;107:700706.

16. Matarasso A. Abdominolipoplasty: A system of classi cation and treatment for combined abdominoplasty and suction- assisted lipectomy. Aesthetic Plast Surg. 1991;15:111-121

17. Nahas, F. X. A pragmatic way to treat abdominal de- formities based on skin and subcutaneous excess. Aesthetic Plast. Surg. 125: 365, 2001.

18. Nahas, F. X. An aesthetic classification of the abdomen based on the myoaponeurotic layer. Plast. Reconstr. Surg. 108: 1787, 2001

19. Nahas, F. X. Advancement of the external oblique muscle flap to improve waistline: A study in cadavers. Plast. Reconstr. Surg. 108: 550, 2001

20. Toranto IR. Resolution of back pain with the wide abdominal rectus plication abdominoplasty. Plast Reconstr Surg. 1988:81:777-779.

21. Toranto IR. The relief of low back pain with the WARP abdominoplasty: A preliminary report. Plast Reconstr Surg. 1990;85:545-555.

22. Gracovetsky S, Farfan H, Helleur C. The abdominal mechanism. Spine. 1985;10(4):317-324.

23. Tesh KM, Dunn JS, Evans JH. The abdominal muscles and vertebral stability. Spine. 1987;12(5) 501-508.

24. Oneal RM, Mulka JP, Shapiro P, Hing D, Cavaliere C. Wide abdominal rectus plication abdominoplasty for the treatment of chronic intractable low back pain. Plast Reconstr Surg. 2011;127:225-231.

25. Temel, M., Türkmen, A. Berberoğlu, O. Improvements in Vertebral-Column Angles and Psychological Metrics After Abdominoplasty With Rectus Plication. Aesth Surg Journal. 2016, Vol 36(5) 577-587

26. Widgerow AD. Abdominoplasty following colostomy. Ann Plast Surg. 1992;298:454-456.

27. Mast BA. Alleviation of urinary incontinence after abdominoplasty. Ann Plast Surg. 1999;42:456-457.

28. Güneren E, Eroğlu L, Koçak I, Uysal OA. Urinary incontinence was improved after abdominoplasty using a very low incision. Plast Reconstr Surg. 1999;104:1582-1584.

29. Solanki NS, Duf eld JA, Dean NR, Morgan RG. The effect of abdominoplasty on urinary incontinence in women. Plast Reconstr Surg. 2010;126:206e-209e

30. Carruthers KH, Kocak E, Hulsen JH, McMahan JD. Improvement in stress urinary incontinence after abdomi- noplasty. Aesthet Surg J. 2014;34:1091-1098.

31. Alastair Taylor, Steven L. Merten, Gavin D. Sandercoe et al. Abdominoplasty Improves Low Back Pain and Urinary Incontinence. Plast. Reconstr. Surg. 2018. 141: 637.

32. Birdsell, D. C., Gavelin, G. E., Kemsley, G. M., and Hein, K. S. Staying power: Absorbable vs. nonabsorbable. Plast. Reconstr. Surg. 68: 742, 1981.

33. Van Uchelen, J. H., Kon, M., and Werker, P. M. The long-term durability of plication of the anterior rectus sheath assessed by ultrasonography. Plast. Reconstr. Surg. 107: 1578, 2001.

34. Nahas, F.X., Ferreira, L.M.,Augusto, S.M.,Ghelfond, C.Long-term follow-up of correction of rectus diastasis. Plast Reconstr Surg. 2005; 115:1736-1741.
35. Elkhatib H, Buddhavarapu SR, Henna H, Kassem W. Ab-dominal musculoaponeuretic system: Magnetic resonance imaging evaluation before and after vertical plication of rectus muscle diastasis in conjunction with lipoabdominoplasty. Plast Reconstr Surg. 2011;128:733e-740e

36. Tadiparthi S, Shokrollahi K, Doyle GS, Fahmy FS. Rectus sheath plication in abdominoplasty: Assessment of its longev- ity and a review of the literature. J Plast Reconstr Aesthet Surg. 2011;65:328-332.

37. Rosen A, Hartman T. Repair of the midline fascial defect in abdominoplasty with long-acting barbed and smooth absorb-able sutures. Aesthet Surg J. 2011;31:668-673.

38. Nahas FX, Ferreira LM, Ely PB, Ghelfond C. Rectus diastasis corrected with absorbable suture: a long-term evaluation. Aesthetic Plast Surg 2011;35(1):43e8

39. Mestak, O., Kullac, R., Mestak, J. Evaluation of the Long-Term Stability of Sheath Plication Using Absorbable Sutures in 51 Patients with Diastasis of the Recti Muscles: An Ultrasonographic Study. Plast Reconstr Surg. 2012. Volume 130, Number 5

40. de Castro EJ, Radwanski HN, Pitanguy I, Nahas F. Long-term ultrasonographic evaluation of midline aponeurotic plication during abdominoplasty. Plast Reconstr Surg. 2013;132(2):333-338.

41. Gama, L.J.M, Barbosa, M.V.J, Czapkowski, A. Single-Layer Plication for Repair of Diastasis Recti: The Most Rapid and Eficcient Technique. Aesthetic Surgery Journal 2017, 1-8

42. Hunter, G. R., Crapo, R. O., Broadbent, T. R., et al. Pulmonary complications following abdominal lipectomy. Plast. Reconstr. Surg. 71: 809, 1983.

43. Jansen, D. A., Kaye, A. D., Banister, R. E., et al. Changes in compliance predict pulmonary morbidity in patients undergoing abdominal plication. Plast. Reconstr. Surg. 103: 2012, 1999.

44. Gilliland,S. Gastroesophageal reflux following male abdominoplasty. Aesthetic Plast. Surg. 20: 527, 1996.

45. Losken, A., Carlson, G. W., Jones, G. E., et al. Significance of intraabdominal compartment pressures following TRAM flap breast reconstruction and the correlation of results. Plast. Reconstr. Surg. 109: 2257, 2002.

46. Losken, A., Carlson, G. W., Tyrone, J. W., et al. The signif-icance of intraabdominal compartment pressure after free versus pedicled TRAM flap breast reconstruction. Plast. Re- constr. Surg. 115: 261, 2005.

47. Al-Basti JB, El-Khatib HA, Taha A, et al. Intraabdominal pressure after full abdominoplasty in obese multiparous patients. Plast Reconstr Surg. 2004;113:2145-2150.

48. Graça Neto L, Araújo LR, Rudy MR, Auersvald LA, Graf R. Intraabdominal pressure in abdominoplasty patients. Aesthet Plast Surg. 2006:30:655-658.

49. Huang GJ, Bajaj AK, Gupta S, et al. Increased intraabdo-minal pressure in abdominoplasty: delineation of risk factors. Plast Reconstr Surg. 2007; 119:1319-1325.

50. Vaca Escobar, B. "Cambios respiratorios postabdominoplastia", Cir. plás.iberolatinoam, 2007, 33 (1): 69.

51. Mercedes S, Núñez M, Díaz O et al. Variabilidad de la presión intrabdominal en pacientes sometidos a abdominoplastia. Cir.plást. Iberolatinoam. 2009. Vol. 35 - No 4. Pag. 261-270

52. Pereira, N. Sciaraffia, C. Danilla, S. Effects of Abdominoplasty on Intra-Abdominal Pressure and Pulmonary Function. Aesthetic Surgery Journal 2016, 1-6.

53. Rodrigues MA, Nahas FX, Reis RP, Ferreira LM. Does Diastasis Width Influence the Variation of the Intra-Abdominal Pressure After Correction of Rectus Diastasis? Aesthet Surg J. 2015:35(5):583-588.

54. Menz P: Pregnancy after abdominoplasty. Plast Reconstr Surg 98:375 $\pm 376,1996$

55. Borman, Huseyin M.D. Pregnancy in the early period after abdominoplasty. Plastic and Reconstructive Surgery. 2002. 396-397

56. Lawrence WT, McDonald HD: Pregnancy after breast reconstruction with a transverse rectus abdominis musculocutaneous flap. Ann Plast Surg 16:354 \pm 355, 1986

57. Chen $L$, Hartramp F CR, Bennett GK: Successful pregnancies following

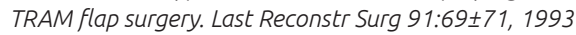

58. Fabio Xerfan Nahas. Pregnancy After Abdominoplasty. Aesth. Plast. Surg. 26:284 $\pm 286,2002$. 